\title{
Author Correction: Altered cerebellar connectivity in autism and cerebellar-mediated rescue of autism-related behaviors in mice
}

Catherine J. Stoodley (1D, Anila M. D’Mello (D), Jacob Ellegood (1), Vikram Jakkamsetti (D), Pei Liu, Mary Beth Nebel(1), Jennifer M. Gibson, Elyza Kelly, Fantao Meng, Christopher A. Cano, Juan M. Pascual, Stewart H. Mostofsky,

Jason P. Lerch and Peter T. Tsai (ID

Correction to: Nature Neuroscience https://doi.org/10.1038/s41593-017-0004-1, published online 30 October 2017.

In the version of this article initially published, the Simons Foundation was missing from the list of sources of support to P.T.T. in the Acknowledgments. The error has been corrected in the HTML and PDF versions of the article.

Published online: 16 March 2018

https://doi.org/10.1038/s41593-018-0096-2

\section{Author Correction: L1-associated genomic regions are deleted in somatic cells of the healthy human brain}

Jennifer A Erwin, Apuã C M Paquola, Tatjana Singer, Iryna Gallina, Mark Novotny, Carolina Quayle, Tracy A Bedrosian, Francisco I A Alves, Cheyenne R Butcher, Joseph R Herdy, Anindita Sarkar, Roger S Lasken, Alysson R Muotri and Fred H Gage

Correction to: Nature Neuroscience https://doi.org/10.1038/nn.4388, published online 12 September 2016.

In the version of this article initially published, NIH grant U01 MH106882 to F.H.G. was missing from the Acknowledgments. The error has been corrected in the HTML and PDF versions of the article.

Published online: 27 April 2018

https://doi.org/10.1038/s41593-018-0131-3

\section{Author Correction: Accumbal D2 cells orchestrate innate risk-avoidance according to orexin signals}

Craig Blomeley, Celia Garau and Denis Burdakov (D)

Correction to: Nature Neuroscience https://doi.org/10.1038/s41593-017-0023-y, published online 27 November 2017.

In the version of this article initially published, a sentence in the third paragraph read, "Suppression of natural orexin signaling by orexin receptor antagonism (Fig. 3b-e) led to increased risk-avoidance behaviors." "Increased" has been changed to "decreased" in this sentence. The error has been corrected in the HTML and PDF versions of the article.

Published online: 5 June 2018

https://doi.org/10.1038/s41593-018-0166-5 